\title{
BUENAS PRÁCTICAS DE ENSEÑANZA DESDE LA CONCEPCIÓN DEL PROFESORADO
}

\section{Ma Fuensanta Martínez Ortiz, José Miguel Nieto Cano y Mónica Vallejo Ruiz}

\section{RESUMEN}

La investigación que aquí presentamos es un estudio descriptivo de las diferentes perspectivas docentes sobre buenas prácticas de enseñanza en el aula, partiendo de tres dimensiones: la identificación y justificación de la buena práctica, la planificación y evaluación de la misma y el desarrollo de ésta en el aula; tomando en cuenta la interacción entre estos procesos y la diferenciación de contextos de enseñanza. Se trata de un estudio realizado a seis docentes (dos maestras de $2^{\circ}$ ciclo de Educación Infantil, dos maestras de Educación Primaria y dos profesores de Educación Secundaria Obligatoria) de la Comunidad Autónoma de la Región de Murcia, utilizando para ello entrevistas semi-estructuradas. A partir del análisis de la información recogida, podemos concluir que existen pequeñas diferencias entre los docentes, en cuanto a cómo identifican, planifican y desarrollan la buena práctica de enseñanza en el aula.

Palabras clave: buenas prácticas, enseñanza de calidad, docentes, enseñanza eficaz, buena enseñanza.

\section{TITLE: GOOD TEACHING PRACTICES FROM TEACHERS' CONCEPTION}

\begin{abstract}
The research presented here is a descriptive study of the different teaching perspectives on good teaching practices in the classroom, based on three dimensions: identification and justification of good practice, planning and evaluation of the same and its development in the classroom; taking into account the interaction between these processes and differentiation of teaching contexts. This is a study on six teachers (two teachers of the 2nd cycle of Early Childhood Education, two Elementary Education teachers and two Secondary Education teachers from the Autonomous Community of the Region of Murcia, using semi-structured interviews. From the analysis of the information gathered, we can conclude that there are small differences among teachers regarding how to identify, plan and develop good practice of classroom teaching.
\end{abstract}

Keywords: Good Practices, Quality Education, Teachers, Effective Teaching, Good Teaching.

Ma Fuensanta Martínez Ortiz. < fuensanti87@hotmail.com>. José Miguel Nieto Cano.<nietos@um.es>. Mónica Vallejo Ruíz.<monicavr@um.es>.Departamento de Didáctica y Organización Escolar. Universidad de Murcia. Original recibido: 14-12-15. Original aceptado: 25-02-16. 


\section{Introducción}

El presente trabajo explora la idea de enseñanza de calidad y su proyección en el aula teniendo en cuenta la perspectiva del profesorado; o lo que es lo mismo, con este trabajo nos interesa conocer en qué consisten aquellas prácticas de enseñanza que los docentes juzgan como "de calidad" y en qué términos valoran (cómo o por qué) las mismas en el escenario particular de su actividad docente. Por consiguiente, los objetivos generales que nos planteamos son los siguientes:

- Identificar buenas prácticas de enseñanza desde el punto de vista del profesor.

- Explorar y comprender la relación que establece el profesor entre la buena práctica de enseñanza y la planificación y evaluación de la misma.

- Analizar y describir el desarrollo de buena práctica de enseñanza en el aula en términos de actividad como de condiciones de la misma.

En este trabajo, adoptamos la concepción de la enseñanza de calidad como la combinación de buena enseñanza y de enseñanza eficaz (Fenstermacher y Richardson, 2005). Aunque el planteamiento de nuestro trabajo centra su atención en la figura del profesor y sus prácticas de enseñanza en el aula, ello no menoscaba en absoluto la importancia de otros factores y condiciones que llegan a afectar a la calidad de las mismas y que están más allá del profesor y de su enseñanza. La buena enseñanza es como indican Porta y Sarasa (2006, p.70) aquella cuyas prácticas docentes son capaces de provocar acciones y cambios en los estudiantes con un sentido moral, dándole más importancia al aprendizaje y al compromiso del profesor, que transmitir una serie de conceptos de manera lineal. Allí es donde reside la importancia del concepto de las "buenas prácticas" (p.70).

En este sentido, abordamos las buenas prácticas como tópico general de estudio, las cuales han adquirido en las últimas décadas gran relevancia en el ámbito de la educación. Nuestro interés descriptivo se centra en tres dimensiones:

- Identificación y justificación de la buena práctica de enseñanza desde el punto de vista del docente.

- Planificación y evaluación de la buena práctica desde el punto de vista del docente.

- Desarrollo de la buena práctica de enseñanza en el aula en términos de actividad como de condiciones de la misma. 
A partir de dichas dimensiones podremos tener una imagen comprehensiva de nuestro objeto de estudio, las "buenas prácticas de enseñanza”, y que a su vez constituyen los objetivos generales ya mencionados. Dimensiones que además tendrán en cuenta los elementos o aspectos que forman parte del contexto de la práctica docente analizada, esto es, el aula.

\section{Metodología}

Nos encontramos ante un estudio de casos fundamentalmente descriptivo y de corte transversal, propio de la investigación cualitativa que aplica métodos y técnicas de investigación de las Ciencias Sociales al estudio de la Educación por medio, básicamente, de la recogida y análisis de datos no numéricos. Nuestro interés descriptivo, como ya hemos anunciado anteriormente, está centrado en tres dimensiones generales.

La población de interés está constituida por maestras en Educación Infantil ( $2^{\circ}$ ciclo), maestras en Educación Primaria y profesores de Educación Secundaria Obligatoria (en adelante ESO) en la Comunidad Autónoma de la Región de Murcia de cuatro centros educativos públicos distintos, dependiendo del caso estudiado. En este marco de referencia, hemos optado por un muestreo no probabilístico de disponibilidad y estratificado, seleccionando dos maestras de Educación Infantil, dos de Educación Primaria y dos profesores de ESO.

Para este estudio se juzgó pertinente la utilización de la técnica de entrevista semi-estructurada y la observación formal (notas de campo), siguiendo la guía de entrevista y de observación (ver Anexo I). Posteriormente, la información recogida (notas de campo y grabaciones de audio) fue analizada mediante el análisis de contenido temático (Krippendorff, 2013; Bardin, 1986). En dicho proceso alternamos la clasificación y reducción del material original, hasta que la evidencia se hace visible, mediante categorías.

\section{Resultados}

Los resultados de nuestro estudio sobre la identificación, planificación y desarrollo de buenas prácticas de enseñanza en el aula en los niveles de Educación Infantil, Primaria y Secundaria Obligatoria, considerados en conjunto, son los siguientes: 
3. I. a) Identificación y justificación de la buena práctica de enseñanza desde el punto de vista del docente.

\section{- Tipologías}

En relación a esta primera dimensión de nuestro objeto de estudio, los docentes entrevistados tienden a identificar una buena práctica (véase Tabla I) con formas de enseñar organizadas a nivel grupal, en su mayoría, que favorecen la interrelación entre el alumnado, ya que estos participan y colaboran en el aprendizaje de determinados contenidos educativos. Este tipo de enseñanza grupal e interactiva podría ser considerada como un factor de enseñanza eficaz según Teddlie y Reynolds (2000), ya que la práctica de enseñanza fomenta la comunicación entre los implicados, favoreciendo así un clima de convivencia en el aula. Además, la identificación que estos docentes hacen también concuerda con la idea de Darling (200I) sobre el "aprendizaje cooperativo", una de las facetas de su modelo de buena enseñanza que se caracteriza por la mejora de la convivencia en el aula gracias a la comunicación. En la siguiente tabla aparecen ocho tipologías de buenas prácticas identificadas por los entrevistados, sin embargo la "creatividad verbal" del caso 3 y el "aprendizaje por refuerzo o recompensa" del caso 5 no fueron observadas en el aula y, por tanto, no son descritas en este estudio.

\begin{tabular}{lcl}
\hline Nivel & Casos & Buenas Prácticas \\
\hline \multirow{2}{*}{ Educación Infantil } & I & Aprendizaje cooperativo \\
\cline { 2 - 3 } & 2 & Aprendizaje a través del juego \\
\hline \multirow{2}{*}{ Educación Primaria } & 3 & $\begin{array}{l}\text { Creatividad verbal } \\
\end{array}$ \\
\cline { 2 - 3 } & 4 & Aprendizaje a través de valores \\
\hline \multirow{2}{*}{ Educación Secundaria musical sincronizada } \\
\cline { 2 - 3 }
\end{tabular}

Tabla I. Tipologías identificadas 
Parecería que los docentes tienden a valorar la calidad de la enseñanza en virtud de que cumpla preceptos de "buena enseñanza", en este caso, relativos a configuraciones de actividad y agrupamientos en el aula que faciliten "supuestamente" el aprendizaje cooperativo del alumnado. Pero, ¿realmente es así? ¿Cómo lo saben? Nuestros participantes, al menos, tienden a coincidir en esa apreciación. Estudios como el de González González (20I0) sobre "El alumno ante la escuela y su propio aprendizaje" apoya esa expectativa por la disposición y comportamiento observable en él a través de su atención e implicación, promoviendo así el enganche del alumno con el aprendizaje (p. II).

\section{- Justificación}

Esta categoría centra su atención en por qué consideran como buenas dichas prácticas. Pues bien, el motivo que dan los docentes entrevistados es básicamente por la actitud positiva reflejada en su alumnado durante el desarrollo de la práctica, ya que según éstos mejora su integración y tiene relación con su vivencia personal (Infantil); anima a la participación, motiva a escuchar y tiene un carácter innovador (Primaria); y mejora la autoestima, anima a la participación y supone un aprendizaje significativo dado su constructivismo (Secundaria). De modo que la implicación en las actividades de enseñanza y las tareas académicas conduce a un aprendizaje significativo. Esta idea se corresponde con el "proceso activo" que Darling (200I) considera como otra de las facetas del modelo de buena enseñanza, ya que los alumnos no se limitan solo a escuchar sino a operar mediante su implicación en el desarrollo de la práctica. De cualquier modo, la "bondad" de la práctica en el aula en general, o del aprendizaje cooperativo en particular, se vincula frecuentemente con su "valor motivador" para el alumnado y así, cabe suponer, su previsible impacto (positivo) en el aprendizaje por éste del correspondiente contenido educativo. Dicho impacto nos podría estar indicando la potencialidad que tienen estas prácticas dada la significatividad que tiene para el alumnado, por su implicación y su acción en ella (Marqués, 2002).

Dentro de las tipologías de las buenas prácticas estudiadas, el "aprendizaje cooperativo" se asocia a una metodología activa o centrada en el alumno denominada "aprendizaje a través del juego". De ahí que toda práctica que conlleve dicha cualidad se considere "per se" innovadora y, por ende, buena o eficaz, en su caso. Y no sólo en relación con el aprendizaje de los contenidos educativos, sino también con el aprendizaje de la "colaboración" entre el propio alumnado (interacciones de 
comunicación y participación por medio de las tareas académicas). Por lo demás, los principios "constructivistas", el "aprendizaje significativo" y el "aprendizaje por descubrimiento" están, asimismo, vinculados a la idea de "buena práctica” en el pensamiento y toma de decisiones de los docentes.

\section{- Sentido}

Por otro lado, las formas de enseñar identificadas como buenas se distinguen del resto, según los docentes entrevistados, por el carácter o sentido que les atribuyen, diferente en cada etapa (véase Figura I).

1. Aprendizaje cooperativo

2. Aprendizaje a través del juego

3. Creatividad verbal y aprendizaje a través de valores

4. Lectura musical sincronizada

5. Trabajo en pequeños equipos y aprendizaje por refuerzo o recompensa

6. Aprendizaje por descubrimiento
- Su caracter social

- Su carácter vivencial

- Su carácter participativo

-Su carácter motivador

- Su factor sorpresa

- Su percepción de un verdadero aprendizaje

Figura I. ¿Qué las distingue de otras formas de enseñar? 
Esas características a los que los docentes aluden para distinguir una buena práctica del resto tienen cierta coincidencia con los criterios de identificación de una buena práctica de Ballart (2007), como es el caso de que ésta sea innovadora, sostenible, transferible y participativa. En este sentido, una buena práctica se distingue del resto:

- por ser algo nuevo para el alumnado;

- por el impacto positivo que produce en el aprendizaje de éste;

- por conseguir que las acciones llevadas a cabo en ella constituyan una experiencia social o vivencial para el alumnado;

- y por implicar a éste en el desarrollo de la misma mediante su participación activa.

En definitiva, podemos considerar que lo que la distingue del resto es su capacidad de atraer al alumno a participar dada su novedad y relación con su experiencia personal.

\section{- Condiciones}

Por otra parte, se trata de prácticas que son seleccionadas por los docentes entrevistados en virtud de la percepción de una circunstancia o situación coyuntural que afecta al alumnado en su actitud y en su aprendizaje.

Como podemos observar en la Figura 2, el momento en el que es aplicada la buena práctica va a depender del alumno, de cómo éste se encuentre, y del tipo de contenido que se pretenda dar. De manera que una buena práctica se adecúa al desarrollo personal del alumno, otra de las facetas que sostiene Darling (200I) en su modelo de buena enseñanza. $Y$ es aquí donde podemos apreciar cierta eficacia por parte de los entrevistados, ya que éstos tienen en cuenta las necesidades de su alumnado y quieren solucionarlas con un tipo de prácticas capaz de despertar en su alumnado una actitud motivadora. Es lo que Stronge (2010) considera como una cualidad de la eficacia docente: llevar a cabo una enseñanza basada en las necesidades del alumnado. 


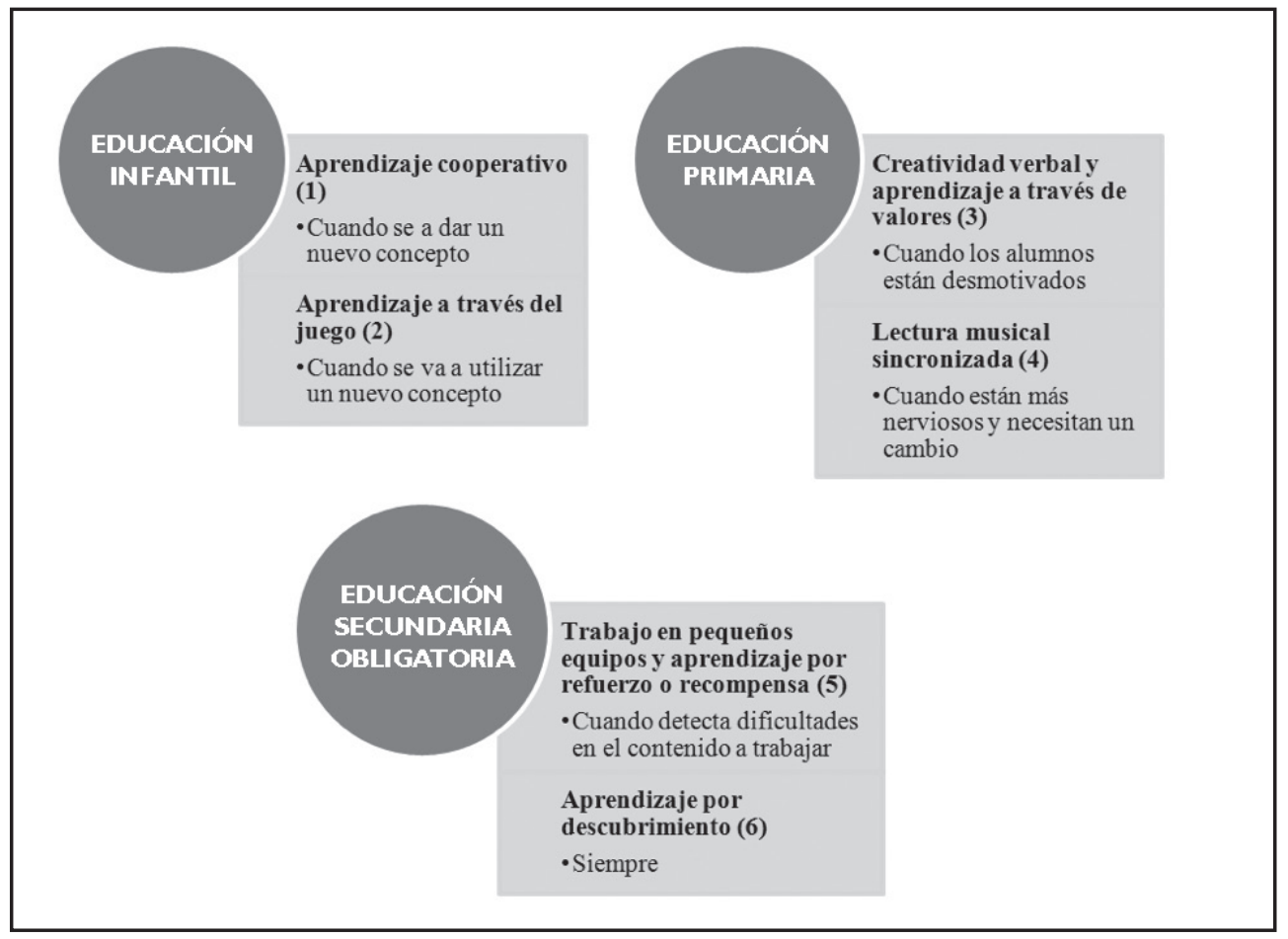

Figura 2. Momento en el que es aplicada la buena práctica

3.2. b) Planificación y evaluación de la buena práctica desde el punto de vista del docente.

\section{- Propósito}

Partiendo de esta segunda dimensión de nuestro objeto de estudio, el propósito de los docentes de llevar a cabo estas formas de enseñar está íntimamente relacionado con las condiciones de aula, haciéndose necesarias por una situación coyuntural, y es a partir de ella cuando éstos se plantean qué han de conseguir para dar solución a esa situación. 


\section{EDUCACIÓN INFANTIL}

Objetiv 0: aprender nuevos conocimientos

Momento: cuando quieren transmitir nuevos conocimientos

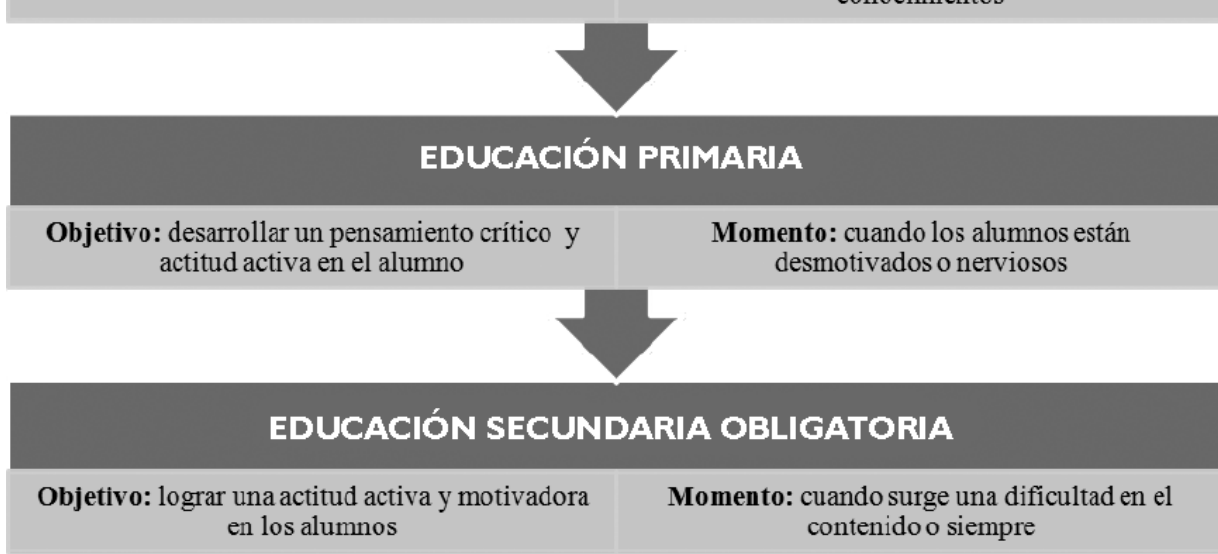

Figura 3. Relación propósitos-momentos

De nuevo podemos observar que el desarrollo de una buena práctica va a depender del alumno, de cómo éste se encuentre, y del tipo de contenido que se pretenda dar, adecuándose por tanto al desarrollo de su alumnado.

\section{- Diseño}

Sin embargo, la planificación de las ocho buenas prácticas identificadas no siempre es abordada de la misma manera por los docentes entrevistados, ya que la planificación de dichas prácticas presenta dos posturas o situaciones distintas:

- Por un lado, se plantean sobre la marcha en función de las condiciones de aula. En este caso no son objeto de planificación formal, sino más bien un tipo de preparación, ya que la práctica es espontánea y natural (sin libro), como es el "aprendizaje 
cooperativo" (caso I), "aprendizaje a través de valores" (caso 3) y "trabajo en pequeños equipos o aprendizaje por refuerzo o recompensa" (caso 5).

- Y por otro lado, es una planificación previa y prevista en la programación docente anual de forma prescriptiva (formal, racional, con el libro), aunque en ocasiones flexibles, como es el "aprendizaje a través del juego" (caso 2), "lectura musical sincronizada" (caso 4) y "aprendizaje por descubrimiento" (caso 6).

En este sentido, consideramos que el tipo de planificación no depende de la etapa educativa, sino más bien de la aptitud y características del profesor: aquel que anticipa sus inquietudes en relación al desarrollo de la enseñanza (comunicación, la reacción del alumnado, el tiempo que va a requerir, etc.) y los factores que pueden favorecer su buen funcionamiento; en contraposición con aquel que suele recurrir a la improvisación, aprovechando una situación coyuntural para aplicarlas; entendiendo que éstas siempre causan resultados positivos en el aprendizaje de los alumnos.

Teniendo en cuenta el "nivel" de planificación, el diseño de la actividad de aula se correspondería más bien, con un formato de "lección" o incluso de "unidad didáctica", en todo caso más cercano al día a día de la interacción didáctica entre docentes y alumnado en el aula, sobre unos contenidos determinados, que a la previsión formal de la programación docente en el curso y área curricular. Sí que es cierto, como sostiene Marqués (2002), que estos docentes tienen en cuenta antes de aplicar su práctica las características individuales y grupales de los alumnos (necesidades, intereses, etc.) y, a partir de ellas, se plantea unos propósitos para darles respuesta, mediante formas de enseñar participativas y colaborativas. Esa preocupación por las características de los alumnos es lo que Bain (2004) considera como un rasgo de eficacia docente.

\section{- Revisión}

Por otro lado, dichas prácticas suelen ser revisadas por los entrevistados al finalizar la intervención, cuestionándose los docentes si lo están haciendo bien o no, con el fin de mejorar futuras implementaciones. Es lo que Marqués (2002) considera como una de las pautas que se han de llevar a cabo en el desarrollo de una buena práctica, el momento postactivo. Para los entrevistados, las formas de enseñanza consideradas como buenas pueden ir cambiando, tras revisarlas, en algún detalle año tras año con ánimo de mejorarlas, o más bien, adaptarlas a circunstancias del curso/grupo (nivel madurativo del alumnado, habilidad para colaborar). Además, se contemplan 
cambios en el uso de materiales o recursos de apoyo al desarrollo de la práctica en el aula y que son confundidos, en la mayoría de los casos, por adaptaciones. Tan sólo se menciona una adaptación curricular significativa en la etapa de Educación Secundaria Obligatoria, al adaptar el contenido al nivel de un ACNEE. A pesar de no haber realizado estos docentes adaptaciones significativas en sus prácticas, sí parece quedar clara su intención por mejorarlas mediante pequeños cambios.

\section{- Repercusión}

Desde la perspectiva de los docentes entrevistados, la repercusión que tiene el desarrollo de su actividad en el aprendizaje de su alumnado dicen ser positiva, independientemente de la etapa educativa, por el efecto que tiene en el alumnado: una actitud motivadora y de gran interés. Esto nos remite de nuevo a por qué consideran nuestros casos dichas prácticas como "buenas".

Para evaluar o valorar la repercusión que tiene la buena práctica en el alumnado, la observación es la técnica más usada por los docentes, especialmente en Infantil, ya que dicha técnica les permite observar las acciones e interacciones del alumnado en el aula. Pero además de ésta, los entrevistados utilizan otra forma de evaluar: la supervisión del trabajo del alumnado (Primaria) y la evaluación por competencias (caso 5). Sin embargo, desconocemos si el desarrollo de estas buenas prácticas influye en las calificaciones del alumnado o si ese efecto positivo perdura o se extingue una vez finalizada la sesión. Tan sólo conocemos el efecto que tiene en el alumnado en el momento en el que se lleva a cabo la actividad en el aula y cómo se evalúa el aprendizaje en ese momento.

En la siguiente tabla podemos ver algunos indicadores que sustentan esa repercusión positiva en el aprendizaje del alumno, mencionados por los entrevistados. 


\begin{tabular}{|c|c|c|}
\hline Casos & Tipos & Repercusión Positiva \\
\hline \multirow{2}{*}{ I } & “Aprendizaje & - Mayor atención \\
\hline & Cooperativo" & - Mayor imaginación y creatividad \\
\hline \multirow{2}{*}{2} & \multirow{2}{*}{$\begin{array}{l}\text { "Aprendizaje a través del } \\
\text { juego" }\end{array}$} & - Mayor motivación (protagonistas). \\
\hline & & - Vivencial (lo aplican a la vida diaria). \\
\hline \multirow{3}{*}{3} & "Creatividad verbal" & - Mayor atención. \\
\hline & \multirow{2}{*}{$\begin{array}{l}\text { "Aprendizaje a través de } \\
\text { valores" }\end{array}$} & - Vivencial (lo aplican a la vida diaria) \\
\hline & & - Mayor atención \\
\hline \multirow{3}{*}{4} & \multirow{3}{*}{$\begin{array}{l}\text { "Lectura musical } \\
\text { sincronizada" }\end{array}$} & - Mayor motivación. \\
\hline & & - Mayor atención. \\
\hline & & - Mayor cercanía \\
\hline \multirow{3}{*}{5} & & - Refuerzo positivo en el estado anímico. \\
\hline & $\begin{array}{l}\text { "Aprendizaje por } \\
\text { refuerzo o recompensas" }\end{array}$ & $\begin{array}{l}\text { - Son conscientes de lo que saben. } \\
\text { - Se sienten capaces de transmitir lo que } \\
\text { saben }\end{array}$ \\
\hline & $\begin{array}{l}\text { "Trabajo en pequeños } \\
\text { equipos" }\end{array}$ & - Aprendizaje significativo. \\
\hline \multirow{3}{*}{6} & \multirow{3}{*}{$\begin{array}{l}\text { "Aprendizaje por } \\
\text { descubrimiento" }\end{array}$} & - Mayor interés por ir a clase. \\
\hline & & $\begin{array}{l}\text { - Quedan atrapados por aquellas situaciones } \\
\text { que son un reto para ellos. }\end{array}$ \\
\hline & & - Temas de interés para el alumno. \\
\hline
\end{tabular}

Tabla 2. Indicadores de la Repercusión en el aprendizaje de los alumnos

Como observamos, el desarrollo de buenas prácticas conlleva que los alumnos desde el momento en el que se inician éstas se sienten atraídos, participando activamente, disfrutando de lo que hacen, aprendiendo nuevos conocimientos en relación al día a día, sintiéndose capaces de hacer frente a nuevas situaciones dada su seguridad, entre otros aspectos. Aun así, si la mayoría de los casos afirma tener una repercusión positiva en el aprendizaje del alumnado, ipor qué suelen revisar sus 
prácticas? Una de las respuestas a esta cuestión sería, desde nuestro punto de vista, el interés de nuestros casos por mejorar y perfeccionar el proceso de enseñanza y aprendizaje. Es aquí donde la práctica se transforma en praxis, orientada ésta hacia la mejora (Zabalza, 20I2).

3.3. c) Desarrollo de la buena práctica de enseñanza en el aula en términos de actividad como de condiciones de la misma.

- Proceso de implementación.

Y finalmente, la tercera dimensión de nuestro objeto de estudio está centrada en el desarrollo de 6 prácticas observadas en el aula de las ocho descritas, quedando sin observar la "creatividad verbal” identificada por el caso 3 (Primaria) y el "aprendizaje por refuerzo o recompensa" identificada por el caso 5 (ESO). Centrándonos en las 6 formas de enseñar observadas, hemos podido observar que todas ellas conllevan trabajo en grupo en el aula, pero cada una con un contenido y un formato diferente (lúdico, preguntas, reproducciones, etc.). Las prácticas que hemos observado en el aula denotan ciertas cualidades comunes en todos los casos que Kyriacou (1997) consideraba como eficacia docente: explicación clara de la actividad, fomento de la participación e implicación, supervisión del trabajo, organización de la actividad, fomento del aprendizaje constructivista, logro de objetivos y utilización de preguntas al aire para ser respondidas en el momento.

Asimismo, hemos observado en el desarrollo de esas buenas prácticas expectativas de los docentes en relación a la capacidad que tiene el alumnado para llevar a cabo la actividad con éxito, lo cual le motiva a participar con mayor entusiasmo y confianza. Esto es lo que Baños (2010) y Rodríguez Izquierdo (2006) denominan efecto "Pigmalion", considerado éste como elemento de eficacia al transmitir dichas expectativas al alumnado. En este tipo de prácticas, hemos observado como el alumnado participa y colabora en su desarrollo (ya sea en grupo o de manera individual) en un entorno motivador, dadas las expectativas que tiene el docente sobre él, por el trato igualitario que se les da y por tener en cuenta sus intereses (uno de los indicadores de potencialidad didáctica). Sin embargo, son prácticas que dependiendo de la etapa en la que nos encontremos presentan un tipo de comunicación más cercana y cálida que otras.

Tomando en cuenta el proceso de implementación, las buenas prácticas observadas suelen "comenzar" con la presentación de nuevos contenidos relacionados con los 
ya dados, aunque se diferencian en el cómo la llevan a cabo: mediante una asamblea y lectura de un cuento (Infantil) o mediante la explicación de la actividad a desarrollar y normas a respetar (Primaria y ESO). El "desarrollo" de éstas suelen hacerse con formulaciones de preguntas por parte del docente a las que son respondidas de manera inmediata por el alumnado, siendo esto una cualidad de eficacia docente (Kyriacou, 1997). Estas intervenciones ayudan a que cada alumno vaya construyendo su propio aprendizaje. Sin embargo, en dicho desarrollo encontramos también diferencias según el enfoque (cooperativo, lúdico, trabajo en cadena y crítico) y el contenido (primavera, letras, valores, figuras musicales, medidas de tendencia central, etc.) que nuestros casos le dan a su práctica, a pesar de ser todas ellas grupales. Además, en esta fase de intervención se producen interacciones lineales (explicaciones o preguntas) y en red (trabajo en grupo o tema de discusión) (Marqués, 2002). Y aunque la forma de "cerrarlas" es dispar en cada caso, la mayoría finaliza con una puesta en común y la recogida del material utilizado, en una sola sesión.

\section{- Contenido}

Además del proceso de implementación (inicio, desarrollo y cierre), se han analizado factores y condiciones en las que son desarrolladas las buenas prácticas identificadas y observadas. Uno de los factores a los que hacemos alusión es referido a la complejidad del contenido educativo. En este sentido, hemos observado como nuestros casos tienden a recurrir a su "buena práctica" cuando prevén que el alumnado va a manifestar dificultades en el aprendizaje del contenido en cuestión (particularmente en las etapas de Educación Primaria y ESO) o cuando quieren incorporar nuevos contenidos (Infantil).

\section{- Roles}

Otra de las categorías que hemos utilizado para analizar la información recogida en las entrevistas y observaciones es la función o rol que desempeña tanto el profesor como el alumno en el desarrollo de la buena práctica. En dichas prácticas el docente suele ser el encargado de facilitar la información, controlar la situación, guiar y motivar a su propio alumnado para que participe. Esto nos recuerda al concepto de "andamiaje" al que hacen referencia MacNaughton y Williams (2009), en el que el docente es el encargado de proporcionar un apoyo o ayuda al alumnado mientras que éste está desarrollando su tarea. 


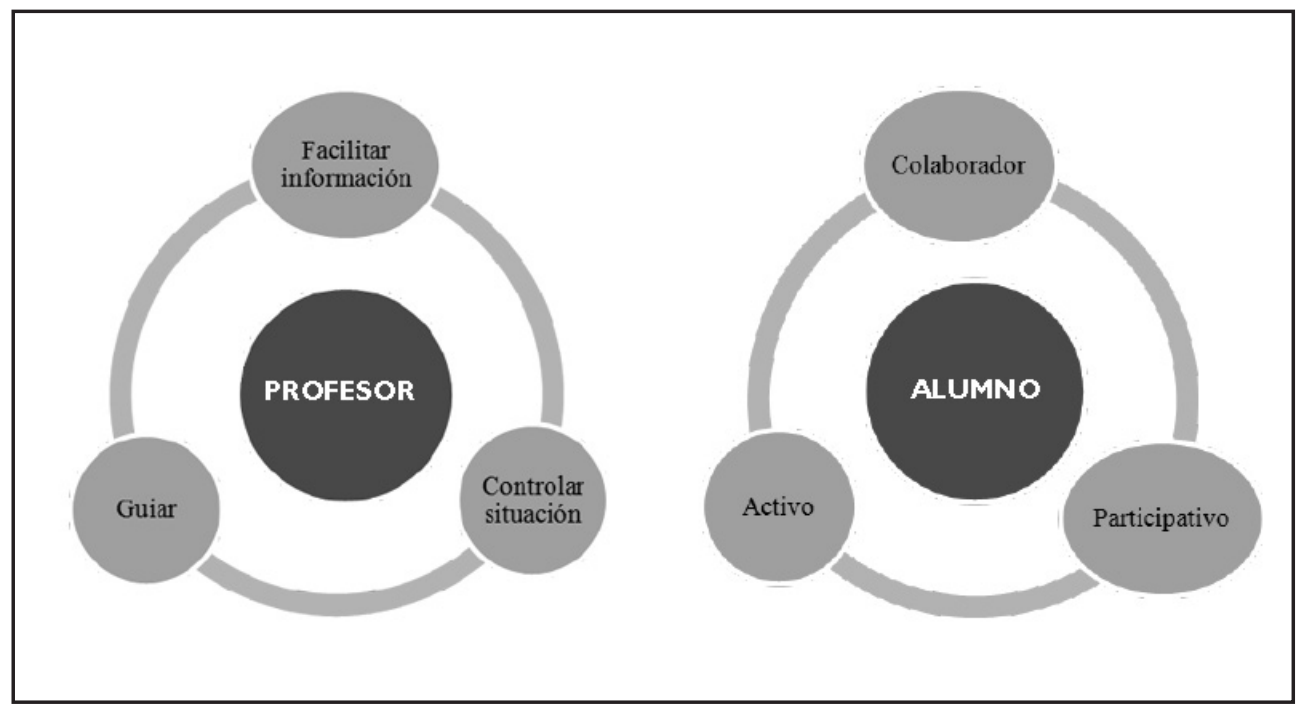

Figura 4. Roles del docente y del alumno

Por otro lado, hemos observado como el alumnado, en este tipo de situaciones, se muestra activo, colaborativo y participativo en las tareas académicas, lo cual contribuye a crear un clima en el aula de diálogo y de intercambio de opiniones entre los propios compañeros tanto en el pequeño como en el gran grupo, favoreciendo así que el aprendizaje sea más significativo para éstos. Esto se corresponde con los principios generales que Biggs (2006) establece sobre buena enseñanza (conocimientos estructurados, contexto motivador, la actividad del alumno y la interacción con los demás). Además, consideramos que estos roles pueden producir en el alumnado lo que Cabrera y De la Herrán (2010) denominan aprendizaje dialógico y aprendizaje cooperativo.

\section{- Recursos}

A lo largo del proceso de implementación de las seis buenas prácticas, hemos observado que los materiales utilizados son aquellos que suelen estar disponibles, habitualmente, en un aula (colores, pegatinas, pizarra tradicional...), recurriendo en ocasiones a otros (videos, P.D.I., etc.). Son estos últimos los que despiertan en el alumnado mayor interés y motivación por las características y formato que tienen. 
- Organización de los alumnos y normas de clase

En cuanto a la organización de los alumnos, éstos se encuentran normalmente organizados en grupos, aunque dependiendo de la fase en la que se encuentre la actividad, éstos trabajan sucesivamente de manera individual, en pequeño $\circ$ en gran grupo. Dicha organización es realizada por los docentes al principio de curso o en el instante en el que se va a realizar la buena práctica, según nos han transmitido cuando han sido entrevistados.

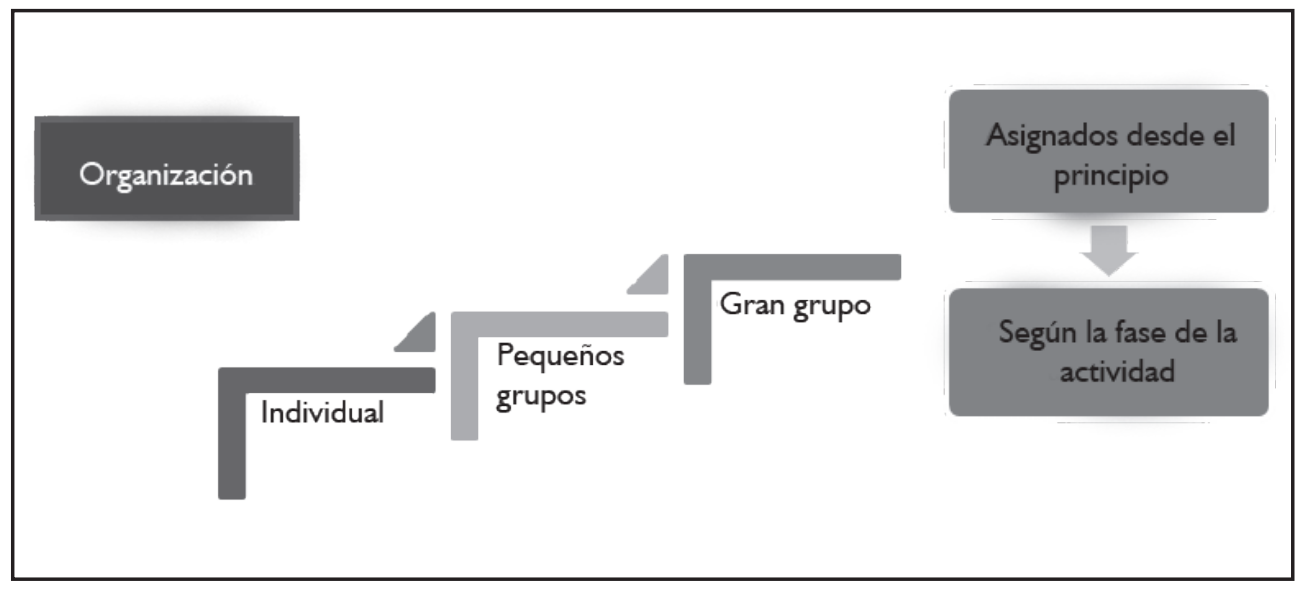

Figura 5. Organización de los alumnos

Asimismo, según los docentes hay establecido un sistema de normas que regula básicamente el orden y silencio dentro del aula durante el desarrollo de la práctica y que hemos podido observar en el aula durante su desarrollo. Esto último es lo que se conoce por "gestión del aula", considerada ésta como una de las cualidades que ha de poseer un profesor eficaz (Kyriacou, 1997; Stronge, 2010). Dicha gestión y los roles que asumen los docentes y el alumnado, remiten a acciones capaces de crear un ambiente adecuado y productivo. Por lo tanto, constituye una actividad de gestión de aula preocupada y centrada en apoyar y facilitar resultados de aprendizaje tanto académicos como socio-emocionales de los alumnos (Weinstein y Weber, 20 I I). Además, hemos podido observar como los docentes especifican el comportamiento que esperan del alumno durante el desarrollo de la práctica, siendo 
esto una destreza del docente según Doyle (2006) y Emmer y Evertson (20I3). Sin embargo, la forma de establecer ese tipo de normas es diferente en algunos casos, al utilizar determinados recursos para ello (una barita para indicar silencio en Infantil o una recompensa si se hace lo acordado en Primaria y Secundaria, por ejemplo).

\section{- Organización espacial y temporal}

Y en relación a cómo se organizan las prácticas estudiadas a nivel espacial y temporal, hemos de afirmar que se tratan de prácticas de corta duración (una sesión aproximadamente) y que son llevadas a cabo en el aula de referencia o específica, sin sufrir modificaciones en el espacio. Además, creemos necesario resaltar que la mayoría de los casos estudiados han sabido gestionar eficazmente el tiempo, comenzando y finalizando las sesiones en el tiempo previsto, así como también el aula, explicando al alumnado la tarea que tiene que hacer en un orden establecido, siendo estos algunos factores que Teddlie y Reynolds (2000) consideran propios de la enseñanza eficaz. Sin embargo, la organización del espacio no es la misma en todos los casos, pues las mesas están organizadas en grupos de cuatro o cinco (Infantil), en forma de " $U$ " en Primaria y en filas o grupos de cuatro o cinco (Secundaria), fomentando así que el alumnado trabaje en grupo y participe en el desarrollo de la práctica, indicadores de potencialidad didáctica y educativa.

\section{Discusión y conclusiones}

¿Podemos hablar de métodos de enseñanza? No está claro, incluso el estudio ha evitado esta categoría para evitar pre-juicios o estereotipos inclinándose por "forma de práctica". De cualquier modo, las prácticas identificadas tal y como son caracterizadas y motivadas por nuestros seis casos remiten a una manera sistemática y recurrente de hacer las cosas en el aula, sentido que, al fin y al cabo, es habitualmente vinculado a la expresión de método.

En definitiva, sea cual fuere la distinción terminológica más acertada, es manifiesta la tendencia a que las "buenas prácticas" combinen elementos de enseñanza o instrucción directa orientada por el docente y elementos de enseñanza basada en el andamiaje (o apoyo) y en la co-construcción, donde aumenta el protagonismo del alumnado al ser creadas condiciones de interacción didáctica que se caracterizan por colaborar, participar, compartir, guiar y ayudar. En este sentido, se da una iniciativa 
y dirección por parte del docente, pero es el alumno el que tiene la oportunidad de desarrollar una conducta activa que le otorgue protagonismo. Los resultados de este trabajo de investigación apuntan la tendencia de una "buena práctica" asociada a un tipo de planificación informal y práctica. Los casos estudiados parten de las "condiciones” en que enseñan, teniéndolas en cuenta: alumnado (sus intereses, actitudes,...), contenidos e incluso metas coyunturales y recursos (materiales, tiempo). A partir de ahí, el docente piensa en actividades o tareas orientadas a involucrar al alumnado; actividades o tareas que permitan, en todo caso, que la enseñanza fluya. Es razonable pensar que tales prácticas, objeto de atención o revisión por nuestros casos, han sido progresivamente elaboradas, de manera rutinaria e incorporadas a su repertorio práctico y que, a posteriori, han sido tomados en cuenta los objetivos o metas para lo que estas actividades podían servir.

\section{Referencias bibliográficas}

Bain, K. (2004). Lo que hacen los mejores profesores de universidad. Valencia: PUV.

Ballart, X. (2007). Proyecto La Mujer Inmigrada. Igualdad, participación y liderazgo en el ámbito local. Guía de buenas prácticas (documento inédito). Barcelona: Universidad Autónoma de Barcelona.

Baños, I. (20 I0). El efecto Pygmalión en el aula. Revista Digital Innovación y Experiencias Educativas, 28, I-9.

Bardin, L. (1986). Análisis de contenido. Madrid: Akal.

Biggs, J. (2006). Calidad del aprendizaje universitario. Madrid: Narcea.

Cabrera, Y. y De la Herrán, A. (2010). Apuntes de pedagogía. La creatividad en la enseñanza. Madrid: CDL.

Darling, L. (200 I). El derecho de aprender. Crear nuevas escuelas para todos. Barcelona: Ariel.

Doyle, W. (2006). Ecological approaches to classroom management. En C. M. Evertson y C. S. Weinstein (Eds.), Handbook of classroom management: research, practice, and contemporary issues (pp. 97-125). Mahwah, NJ: Lawrence Erlbaum Associates. 
Emmer, E. y Evertson, C. (20I3). Classroom management for middle and high school teachers (9a ed.). Boston: Pearson.

Fenstermacher, G. y Richardson, V. (2005). On making determinations of quality in teaching. Teachers College Record, 107 (I), 186-2I3.

González González, M. T. (2010). El alumno entre la escuela y su propio aprendizaje: algunas líneas de investigación en torno al concepto de implicación. Revista Interuniversitaria sobre calidad, eficacia y cambio en Educación, 8 (4), II-3 I.

Kyriacou, C. (1997). Effective teaching in schools: Theory and practice ( $3^{\mathrm{a}}$ ed.) Cheltenham: Nelson Thornes.

Krippendorff, K. (20I3). Content analysis. An introduction to its methodology ( $\left.3^{\mathrm{a}} \mathrm{ed}.\right)$ Los Angeles: SAGE.

MacNaughton, G. y Williams, G. (2009). Teaching young children: choices in theory and practice (2nd ed.). Maidenhead: Open University Press.

Marqués, P. (2002). Buenas prácticas docentes. Recuperado el I7/02/20I 2 de < http:// www.peremarques.net/bpracti.htm\#potencia $>$.

Porta, L. y Sarasa, Ma C. (2006). Concepciones de la buena enseñanza en los relatos docentes: la formación inicial del profesorado de inglés. Revista Praxis Educa, 10, 68-74.

Rodríguez Izquierdo, R. (2006). Éxito y fracaso escolar en contextos socioculturales interculturales: el reto de educar a estudiantes de diverso origen lingüístico y cultural (documento inédito). Sevilla: Universidad Pablo de Olavide.

Stronge, J. H. (20I0). Effective teacher = Student achievement. What the research says? Larchmont, NY: Eye on Education.

Teddlie, C. y Reynolds, D. (Eds.) (2000). The international handbook of school effectiveness research. Londres: Falmer Press.

Weinstein, C. y Weber, W. (2010). Classroom management. En J. M. Cooper (Coord.), Classroom teaching skills (pp. 2I5-25I). Belmont, CA: Wadsworth Cengage Learning.

Zabalza, M. A. (20I2). El estudio de las "buenas prácticas" docentes en la enseñanza universitaria. Revista de Docencia Universitaria, 10 (I), 17-42. 


\section{Anexo I. Protocolo de entrevista}

I. Presentación personal y contextualización del estudio

2. Solicitud y concertación de condiciones de la entrevista

3. Datos de identificación del participante: nivel, sexo, años de experiencia docente

4. Dimensiones y preguntas de indagación:

\section{Desarrollo de}

la enseñanza en

el aula
- Del conjunto de tu actividad docente en el aula, iqué tipos o formas de enseñar destacarías a tu juicio como "buenas prácticas"?

- ¿Por qué/para qué serían "buenas”? (posible fundamento psicopedagógico o criterio que justificaría esa valoración: qué significado tiene para el profesor el calificativo de "buena" o cualesquiera otro que prefiera emplear -como ejemplar, eficaz...-

De esta primera y general pregunta pueden surgir múltiples y diversos tipos o formas de enseñanza, en relación con cada una de las cuales nos interesa particularmente conocer:

Forma I

Forma 2

Forma 3

Forma $\mathrm{N}$

- Su denominación y diferenciación respecto de las otras: icómo se refiere el profesor/a a ella?, ¿qué la distingue de las otras o la hace singular?

- Su intención o sentido curricular e instructivo: ¿cuándo o bajo qué circunstancias se recurre a ella en el aula?

- Su descripción operativa con el mayor detalle posible, es decir su desarrollo como actividad tanto como las condiciones de la misma: ¿en qué consiste?, ¿cómo se lleva a cabo?

- DESCRIPCIÓN DE LA FORMA DE ACTIVIDAD:

- Objetivo ( tipo de intención educativa, resultado de aprendizaje perseguido)

- Contenido (tipo de contenido curricular objeto de enseñanza-aprendizaje)

- Operaciones profesor y operaciones alumnos (roles y acciones de unos y otros utilizando determinados modos de expresión o representación)

- Recursos (materiales o instrumentos que sirven para desarrollar las operaciones)

- Formato (tipo de actividad -individual o grupal- y sistema de normas o reglas que regula el comportamiento y la interacción durante la actividad)

- Tiempo y espacio (duración de actividad y organización espacial para su desarrollo)

Finalmente, interesa conectar cada tipo o forma de actividad en relación con tareas de:

- Planificación

de enseñanza

- Evaluación de

aprendizajes
- ¿La sometes a planificación?, ¿cómo la planificas o programas?

- iHaces algún tipo de adaptación de un curso para otro, o para algún/os alumno/s por alguna causa particular?

- ¿Cómo repercute en el aprendizaje de tus alumnos?, ¿cómo haces la evaluación?

- ¿Sometes esa forma de actividad a revisión o reflexionas sobre ella de vez en cuando?

- ¿La has modificado con el paso del tiempo? 